\title{
Research on Combination of Rough Set and Seeking for Multi-objective Decision-Making
}

\author{
Zhenghua Cui, Jian Huang, Jianguo Hao, Mingguang Gao, and Jiangtao Kong
}

\begin{abstract}
Considering the technical indicator identification problem in preliminary weaponry research, the paper provides a novel approach by combining operational effectiveness with multi-objective optimization algorithms (MOO). Through using effectiveness evaluation approaches based on Rough Set Theory and having the set of $\mathbf{2 0}$ kinds of gunships, which are widely equipped nowadays, as the knowledge base, the paper first evaluates several performance factors and reaches the decision schema satisfying completion requirement after two times of filtering. Then the result is optimized using MOO algorithms under different weights over objectives. During the process, two phrases of filtering are applied, thus reducing the problem complexity. Meanwhile, the function of evaluating is also modified to improve the intuition and accuracy of results. This paper realizes the combinatory application of fuzzy set theory and MOO in the domain of operational effectiveness evaluation.
\end{abstract}

Index Terms-Rough sets, fuzzy multi-objective, configuration schemes, evaluate, decision-making.

\section{INTRODUCTION}

It has numerous of diverse factors with the problem of researching and developing weapons along with the other equipment in advance. On the one hand, the results of operational effectiveness evaluation should meet the requirements of a specific task, on the other hand, the configuration scheme should take restrictions into consideration, such as the situation of the present technology, financial and time aspects. The measure of evaluating the operational effectiveness which is based on rough set theory has advantages to deal with the fuzzy data. In addition, seeking for fuzzy multi-objective decision-making could play an important role in the process of achieve the optimal configuration scheme.

\section{Methods of Evaluating AND SEEKING FOR DECISION-MAKING}

\section{A. Evaluating Operational Effectiveness Based on Rough Sets Theory}

Rough Set theory is proposed by the Polish mathematician Z. Pawlak in 1982, is a new tool to deal with vagueness and uncertainty data [1]. The rough set theory has been found to have quite successful applications in the field of evaluating effectiveness. The primary coverage includes the

Manuscript received January 23, 2015; revised June 2, 2015.

Zhenghua Cui, Jian Huang, Jianguo Hao, and Jiangtao Kong are with the National University of Defense Technology, Changsha, Hunan, China (e-mail: cocolovelife@outlook.com, Huang_jian@139.com, hla2000@tom.com, Jiangtao_Kong@163.com).

Mingguang Gao is with the Beijing Institute of Technology, Beijing, China (e-mail: sunnyangelgmg@outlook.com). interpretation of the connection between the inaccurate data as well as discovering the relationship between objects and attributes [2].

\section{B. Seeking For Fuzzy Multi-objective Decision-Making}

The so-called seeking for fuzzy multi-objective decision-making refers to making decisions in case of multiple objectives that may conflict with each other and are incommensurable with each other. However, in reality, most of the cases are a kind of the multi-objective decision-making problem, for instance, the optimal design of weaponry, combat program evaluation and selection [3] etc. The model could be described as follows:

$$
\max _{x \in X}\{f(x)\}
$$

$X$ is the decision space as well as the feasible region which is determined by characteristics and requirements of actual decision problems. $X$ is the decision variable (while $x \in X$, we always call $X$ the feasible solution).

$$
f(x)=\left(f_{1}(x), f_{2}(x), \cdots, f_{m}(x)\right)^{T}
$$

It represents the vector functions based on the amount of $m, m$ is a positive integer. The vector functions could be benefit type or cost type. According to the actual situation, it just needs to be changed the positive or negative sign.

\section{SAMPLE APPLICATION}

\section{A. Example and Process}

The paper takes the operational effectiveness of military helicopters as the evaluate object and the configuration scheme which we need to confirm as the seeking for fuzzy multi-objective decision-making object. We take advantage of the world's typical top 20 gunships index as the knowledge

\begin{tabular}{|c|c|c|c|c|c|c|c|}
\hline $\mathrm{C} 1$ & C3 & C6 & $\mathrm{C} 7$ & C8 & $\mathrm{C} 9$ & $\mathrm{C} 10$ & $\mathrm{C} 11$ \\
\hline COUNTRY & NAME & LONG & HEIGHT & DIAMETER & POWER & MAXSPEED & SPEEL \\
\hline US & RAH-66 & 14.48 & 3.39 & 11.9 & 3126 & 328 & 305 \\
\hline US & $\mathrm{AH}-64 \mathrm{~A}$ & 17.76 & 4.05 & 14.63 & 1940 & 365 & 265 \\
\hline US & $\mathrm{AH}-64 \mathrm{D}$ & 17.7 & 4 & 14.6 & 1940 & 365 & 265 \\
\hline RU & $\mathrm{K}-50$ & 15 & 4.9 & 14.5 & 4000 & 340 & 290 \\
\hline RU & $\mathrm{K}-52$ & 15 & 4.6 & 14.5 & 4000 & 330 & 279 \\
\hline $\mathrm{CN}$ & Wz-10 & 14.15 & 3.85 & 13 & 3063 & 300 & 270 \\
\hline USA & $\mathrm{AH}-1$ & 13.9 & 4.4 & 14.6 & 2082 & 313 & 278 \\
\hline ZA & CSH-2 & 18.7 & 5.1 & 15.6 & 3808 & 371 & 278 \\
\hline $\mathrm{JP}$ & ${ }_{A H}-1 \mathrm{~J}$ & 13.9 & 4.4 & 14.6 & 1800 & 285 & 227 \\
\hline EU & SA341 & 11.9 & 3.1 & 10.5 & 600 & 310 & 270 \\
\hline EU & PAH-2 & 14 & 4.3 & 13 & 2570 & 320 & 230 \\
\hline RU & $\mathbb{I I}-24$ & 18.5 & 6.5 & 17.6 & 4450 & 335 & 295 \\
\hline IL & TZEFA & 13.8 & 4.4 & 14.6 & 1800 & 290 & 227 \\
\hline $\mathrm{EU}$ & PAH-1 & 8.8 & 3 & 9.8 & 3300 & 242 & 205 \\
\hline EU & A -129 & 14.3 & 3.4 & 11.9 & 1500 & 313 & 240 \\
\hline BR & $500 \mathrm{MD}$ & 7.6 & 2.6 & 8 & 317 & 241 & 221 \\
\hline RU & $\mathbb{M}-17$ & 18.4 & 5.7 & 21.3 & 4000 & 250 & 240 \\
\hline $\mathrm{CN}$ & $W Z-9$ & 13.7 & 4.5 & 11.9 & 1420 & 315 & 280 \\
\hline JP & $\mathrm{CH}-47 \mathrm{~J}$ & 15.5 & 5.7 & $2 * 18.3$ & 4333 & 274 & 259 \\
\hline IL & $\mathrm{UH}-60 \mathrm{~A}$ & 19.5 & 4.8 & 16.5 & 3300 & 296 & 257 \\
\hline $\mathrm{EU}$ & SA330 & 15.5 & 4.92 & 15 & 3500 & 278 & 249 \\
\hline EU & SA321 & 23 & 6.7 & 18.9 & 3150 & 270 & 248 \\
\hline EU & SA319 & 12.8 & 3 & 11 & 700 & 220 & 190 \\
\hline $\mathbb{M Y}$ & LYNX 300 & 13.5 & 3.7 & 12.8 & 1800 & 259 & 225 \\
\hline & & & & & & & \\
\hline
\end{tabular}
base [4]. Partial information of the base is shown in Fig. 1. 
Based on rough set theory and the way of seeking for fuzzy multi-objective decision-making, the use of evaluating operational effectiveness will get the optimal configuration scheme. Assuming the requirement of more than $50 \%$ degree of completion, the process is shown in Fig. 2.

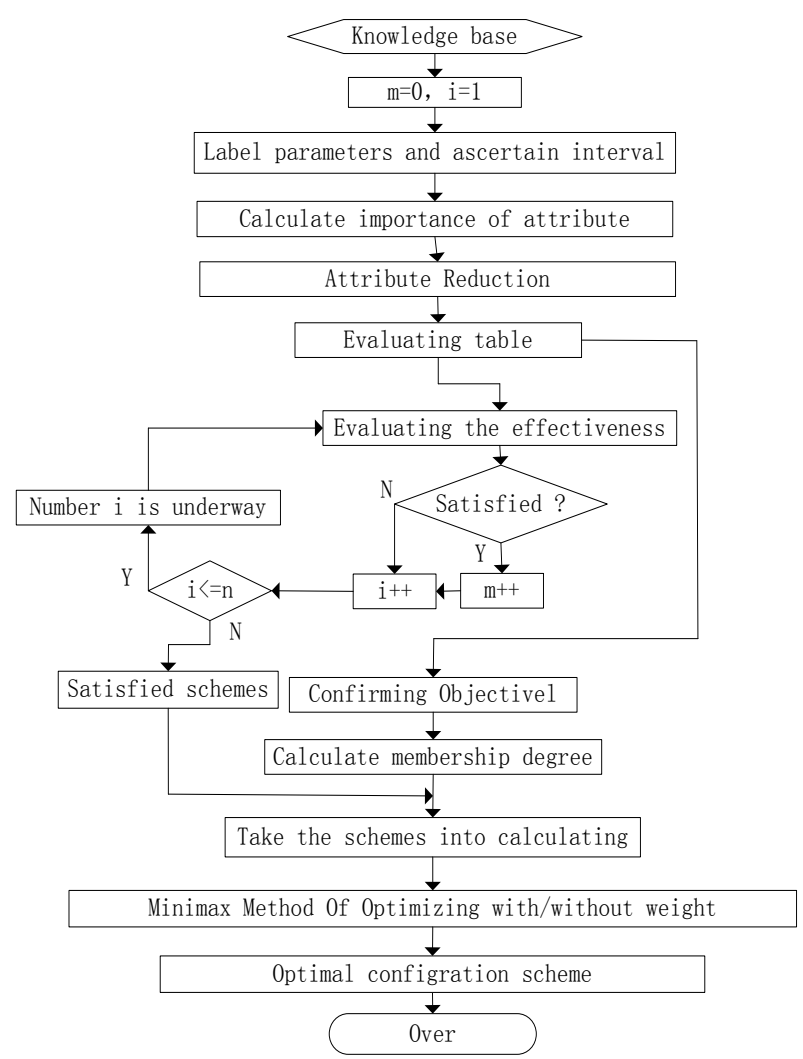

Fig. 2. The operational flowchart.

\section{B. Evaluating Based on Fuzzy Set}

\section{1) Data preprocessing}

Considering the fact that the preprocessing of attribute data [5] isn't the key part of the research, we wouldn't go into details. Through the analysis of rankings of the helicopters from the Internet and combining with the performance parameters above, we can get the comprehensive ranking of the military helicopters. According to the order of the ranking, we divide them into 4 groups with each one possessing 4 helicopters thus representing different effectiveness levels. The effectiveness levels correspond to main intervals of the degree of completion that are listed below:

$$
a:(0.8,1] \text {,b:( 0.8,1 ],c: }(0.8,1], d:(0.8,1]
$$

Selecting height of helicopters, power of engine, maximum rate of climbing and mounting system as objectives to be optimized, we label performance parameters as below:

$$
\text { [High, Middle, Low }]=[a, b, c]
$$

\section{2) Attribute reduction}

The following Table I is a decision table concerning the 20 gunships we mentioned.

$$
U=\left\{X \mid X=x_{i}\right\}(i=1,2, \cdots, 20)
$$

The attribute set is $A=C \mathrm{U} D$ and the condition attribute set is

$$
C=\{\text { Height, Power, Climbing rate, System }\} .
$$

The decision attribute set is

$$
D=\{\text { Effectiveness Level }\} .
$$

TABLE I: EFFECTIVENESS EVALUATING DATA TABLE

\begin{tabular}{|l|l|l|l|l|l|}
\hline$U$ & $C_{7}$ & $C_{9}$ & $C_{12}$ & $C_{18}$ & $D$ \\
\hline$x 1$ & $b$ & $b$ & $b$ & $a$ & $a$ \\
\hline$x 2$ & $b$ & $a$ & $a$ & $a$ & $a$ \\
\hline$x 3$ & $b$ & $a$ & $a$ & $a$ & $a$ \\
\hline$x 4$ & $c$ & $a$ & $c$ & $a$ & $a$ \\
\hline$x 5$ & $b$ & $a$ & $a$ & $a$ & $a$ \\
\hline$x 6$ & $b$ & $a$ & $a$ & $b$ & $b$ \\
\hline$x 7$ & $b$ & $b$ & $a$ & $b$ & $b$ \\
\hline$x 8$ & $c$ & $b$ & $a$ & $b$ & $b$ \\
\hline$x 9$ & $b$ & $b$ & $b$ & $b$ & $b$ \\
\hline$x 10$ & $a$ & $a$ & $a$ & $b$ & $b$ \\
\hline$x 11$ & $a$ & $a$ & $a$ & $b$ & $c$ \\
\hline$x 12$ & $c$ & $c$ & $a$ & $b$ & $c$ \\
\hline$x 13$ & $b$ & $b$ & $b$ & $b$ & $c$ \\
\hline$x 14$ & $c$ & $a$ & $c$ & $b$ & $c$ \\
\hline$x 15$ & $c$ & $c$ & $b$ & $b$ & $c$ \\
\hline$x 16$ & $a$ & $a$ & $b$ & $b$ & $d$ \\
\hline$x 17$ & $b$ & $a$ & $b$ & $c$ & $d$ \\
\hline$x 18$ & $b$ & $a$ & $c$ & $c$ & $d$ \\
\hline$x 19$ & $a$ & $a$ & $c$ & $c$ & $d$ \\
\hline
\end{tabular}

In Table I $C_{7}=$ Height, $C_{9}=$ Power, $C_{12}=$ Climbing rate, $C_{18}=$ System, $D=$ Effectiveness Level .

Divide $U$ as required, and then we could get each positive region of $D[6]$.

$$
\begin{gathered}
\operatorname{POS}_{C}(D) ; \\
\operatorname{POS}_{\left(C-\left\{c_{7}\right\}\right)}(D) ; \\
\operatorname{POS}_{\left(C-\left\{c_{9}\right\}\right)}(D) ; \\
\operatorname{POS}_{\left(C-\left\{c_{12}\right\}\right)}(D) ; \\
\operatorname{POS}_{\left(C-\left\{c_{18}\right\}\right)}(D)
\end{gathered}
$$

Calculate the degree of the importance of parameters $C$ to the effectiveness level $D$.

$$
\gamma_{C}(D)=\frac{\left|\operatorname{POS}_{C}(D)\right|}{|U|}=0.9
$$

Calculate the importance of effectiveness level of each condition attribute $C_{i}$.

$$
\begin{aligned}
& \sigma_{C_{7} D}\left(C_{7}\right)=\gamma_{C}(D)-\gamma_{C-\left\{C_{7}\right\}}(D)=0 \\
& \sigma_{C_{9} D}\left(C_{9}\right)=\gamma_{C}(D)-\gamma_{C-\left\{C_{9}\right\}}(D)=0.2 \\
& \sigma_{C_{12} D}\left(C_{12}\right)=\gamma_{C}(D)-\gamma_{C-\left\{C_{12}\right\}}(D)=0.2 \\
& \sigma_{C_{18} D}\left(C_{18}\right)=\gamma_{C}(D)-\gamma_{C-\left\{C_{18}\right\}}(D)=0.35
\end{aligned}
$$

Normalize the degree of importance $\sigma$, and then we could get:

$$
\begin{aligned}
& \lambda_{7}=0 \\
& \lambda_{9}=0.267 \\
& \lambda_{12}=0.267 \\
& \lambda_{18}=0.467
\end{aligned}
$$

Obviously, $C_{7}$ which represents the height of helicopters should be removed. That is, Power, Maximum rate of climbing and system have more contribution to the effectiveness level.

\section{3) Rule reasoning and initial screening}

Reasoning the information that has been processed, then 
we can get:

$$
\begin{aligned}
F_{1}= & (\{a(0.6, b(0.4)\} ; \\
& \{a(0.6), b(0.2), c(0.2)\} ; \\
& \{a(1)\} ;) \\
F_{2}= & (\{a(0.2), b(0.6), c(0.2)\} ; \\
& \{a(0.8), b(0.2)\} ; \\
& \{b(1)\} ;) \\
F_{3}= & (\{a(0.2), b(0.2), c(0.6)\} ; \\
& \{a(0.4), b(0.4), c(0.2)\} ; \\
& \{b(1)\} ;) \\
F_{4}= & (\{a(0.2), b(0.6), c(0.2)\} ; \\
& \{b(0.4), c(0.6)\} ; \\
& \{b(0.2), c(0.8\} ;)
\end{aligned}
$$

According to the 20 groups of scheme, which have already appeared the attribute sets, we begin initial screening by using the remaining 14 groups of schemes do not appear in the sets. Thus it is obvious of the effectiveness degree of group $(b, b, b)$ as $c$; the effectiveness degree of group $(c, b, c)$ and $(c, c, b)$ as $c$ or less than $c$. Hence, the result of initial filtering is shown in Table II.

TABLE II: INITIAL SCREENING TABLE
\begin{tabular}{|c|l|c|c|}
\hline No. & Group & Remove? & Reason \\
\hline 14 & $(a, b, a)$ & $\times$ & Can't Judge \\
\hline 15 & $(b, a, a)$ & $\times$ & Can't Judge \\
\hline 16 & $(a, a, c)$ & $\times$ & Can't Judge \\
\hline 17 & $(a, c, a)$ & $\times$ & Can't Judge \\
\hline 18 & $(c, a, a)$ & $\times$ & Can't Judge \\
\hline 19 & $(a, b, c)$ & $\sqrt{ }$ & By Group 3 \\
\hline 20 & $(a, c, b)$ & $\sqrt{ }$ & By Group 3 \\
\hline 21 & $(b, a, c)$ & $\times$ & Can't Judge \\
\hline 22 & $(c, b, a)$ & $\times$ & Can't Judge \\
\hline 23 & $(a, c, c)$ & $\sqrt{ }$ & By Group 3 \\
\hline 24 & $(c, a, c)$ & $\times$ & Can't Judge \\
\hline 25 & $(c, c, a)$ & $\times$ & Can't Judge \\
\hline 26 & $(c, b, c)$ & $\sqrt{ }$ & By Group 12 \\
\hline 27 & $(c, c, b)$ & $\sqrt{ }$ & By Group 12 \\
\hline
\end{tabular}

After initial filtering, the remained groups, with the number of 14-18, 21-22 and 24-25, would be disposed by the rule reasoning. Calculate $D\left(F_{i} \mid X_{j}\right)$. For example, the result of the calculation is as follows when $j$ is 14 :

$$
\begin{aligned}
& D\left(F_{1} \mid X_{14}\right)=0.600 ; \\
& D\left(F_{2} \mid X_{14}\right)=0.133 ; \\
& D\left(F_{3} \mid X_{14}\right)=0.200 ; \\
& D\left(F_{4} \mid X_{14}\right)=0.200 .
\end{aligned}
$$

Therefore, we can get the result of the effectiveness evaluation [7]. That is, when the power of the engine is more than 3000 Horsepower, the maximum rate of climbing is between 450 and 590 meter per minute and the capability of the system is strong, the effectiveness degree of fighting of the military helicopter will achieve " $a$ " at $60 \%$ confidence level, the degree will achieve " $b$ " at $13.3 \%$ confidence level, the degree will achieve " $c$ " at $20 \%$ confidence level and the degree will achieve " $d$ " at $20 \%$ confidence level.

\section{4) Improvement and secondary screening}

The above effectiveness degree and the confidence level can only give the range of evaluating result which shows the weakness, less intuitive and imprecise. In this paper, we improve a little bit in this aspect. Use the effectiveness degree and confidence level to acquire more accurate and intuitive specific values. In fact, select the information from the group with factor $j$ as $1,2,3,6,7$, and 8 as the partial knowledge case for correcting.

$$
\begin{array}{ll}
j=1: & \delta_{1}=1.9868 \\
j=2: & \delta_{2}=1.7206 \\
j=3: & \delta_{3}=1.2255 \\
j=6: & \delta_{6}=1.3043 \\
j=7: & \delta_{7}=1.4845 \\
j=8: & \delta_{8}=0.9445
\end{array}
$$

The final completion and the average corrected coefficient are:

Final completion $=\delta / \bar{\delta}$

$$
\bar{\delta}=\frac{1}{6} \sum_{i=1}^{6} \delta_{i}=1.39451
$$

TABLE III: EFFECTIVENESS EVALUATING TABLE

\begin{tabular}{|c|c|c|}
\hline No. & Completion & Satisfied \\
\hline 14 & 0.514 & $\sqrt{ }$ \\
\hline 15 & 0.713 & $\sqrt{ }$ \\
\hline 16 & 0.487 & $\times$ \\
\hline 17 & 0.471 & $\times$ \\
\hline 18 & 0.588 & $\sqrt{ }$ \\
\hline 21 & 0.516 & $\sqrt{ }$ \\
\hline 22 & 0.418 & $\times$ \\
\hline 24 & 0.392 & $\times$ \\
\hline 25 & 0.376 & $\times$ \\
\hline
\end{tabular}

Therefore when $j=14$, the completion is 0.541 , which has already been corrected instead of the original 0.71645 . And the result should satisfy the requirement with no less than $50 \%$ completion. For other 8 groups, repeat the steps above. Results are shown in Table III.

Through secondary filtering, the configuration scheme groups satisfying the above requirement are listed in Table IV.

TABLE IV: SECONDARY SCREENING RESULT

\begin{tabular}{|l|l|}
\hline No. & Scheme Group \\
\hline 14 & $(a, b, a)$ \\
\hline 15 & $(b, a, a)$ \\
\hline 18 & $(c, a, a)$ \\
\hline 21 & $(b, a, c)$ \\
\hline
\end{tabular}

\section{Multi-objective Decision-Making}

1) Confirming the decision objective

In reality, most of cases belong to the multi-objective decision-making problem [8]. When researching the development plan of the military helicopters, the researchers are customarily concerned with various factors like motor performance, capability of the weapons, viability, research costs and time etc. In this paper, besides factors that have been mentioned above, we take cost and time of the research, that are cost type objectives, into consideration. According to the actual situation, we take the power of the engine $\left(f_{1}\right)$, the maximum rate of climbing $\left(f_{2}\right)$, the capability of the system $\left(f_{3}\right)$, research costs $\left(f_{4}\right)$ and time $\left(f_{5}\right)$ as the factors that are closely related to the effectiveness value. 


\section{2) Minimax method of optimizing}

The minimax method of optimizing, with an advantage of good usability, lays its stress on being conservative. The principle is that if $x_{j^{*}} \in X$ satisfies

$$
\mu_{i^{*} j^{*}}=\max _{1 \leq j \leq n} \min _{1 \leq i \leq m}\left\{\mu_{i j}\right\}
$$

$x_{j^{*}}$ is the most suitable scheme. When there are more than two schemes that are satisfied with the formula, all these schemes are tied for the first in suitable. Remove $x_{j^{*}}$ from $X$. Repeat the process so that we can get the ranking of the schemes.

The most suitable scheme which is sought by minimax method of optimizing can ensure the worst objective function to value of the largest. Obviously, it is a conservative method that can avoid making mistakes in game.

$$
F=\begin{gathered}
x_{1} \\
f_{1} \\
f_{2} \\
f_{3} \\
f_{4} \\
f_{5}
\end{gathered}\left[\begin{array}{cccc}
3126 & x_{2} & x_{3} & x_{4} \\
480 & 750 & 760 & 1700 \\
\text { Strong } & \text { Strong } & \text { Strong } & \text { Weak } \\
\text { High } & \text { Middle } & \text { Middle } & \text { Low } \\
\text { Longer } & \text { Long } & \text { Middle } & \text { Short }
\end{array}\right]
$$

Take caution that the evaluations of factors $f_{3}, f_{4}, f_{5}$ are fuzzy. That is, we use natural language [9] to describe the values of schemes, for examples:

("Strong", "Middle", "Weak")-(1,0.7,0.3), ("High", "Middle", "Low")-(1,0.7,0.3) and ("Longer", "Long", "Middle", "Short")-(1,0.75,0.5,0.25). So that $F$ could be translated into the relative membership degree matrix of the objectives. We know that $f_{1}, f_{2}$ and $f_{3}$ are benefit type objectives, while $f_{4}$ and $f_{5}$ are cost type objectives, the relative membership functions are:

$$
\begin{gathered}
\mu_{i j}=\left(f_{i j} / f_{\text {imax }}\right)^{p_{i}}\left(i \in 0^{\text {Benefit type }}\right) \\
\mu_{i j}=\left\{\begin{array}{ll}
1-\left(f_{i j} / f_{i \text { max }}\right)^{p_{i}} & \left(f_{\text {imin }}=0\right) \\
\left(f_{\text {imin }} / f_{i j}\right)^{p_{i}} & \left(f_{\text {imin }} \neq 0\right)
\end{array} \quad\left(i \in 0^{\text {Cost type }}\right)\right.
\end{gathered}
$$

Thus, we can get the updated matrix $F^{\prime}$ and $\mu$.

$$
\begin{aligned}
& \begin{array}{llll}
x_{1}^{\prime} & x_{2}^{\prime} & x_{3}^{\prime} & x_{4}^{\prime}
\end{array} \\
& F^{\prime}=\begin{array}{c}
f_{1} \\
f_{2} \\
f_{3} \\
f_{4} \\
f_{5}
\end{array}\left[\begin{array}{cccc}
3126 & 2082 & 760 & 1700 \\
480 & 750 & 600 & 670 \\
1 & 1 & 1 & 0.3 \\
1 & 0.7 & 0.7 & 0.3 \\
1 & 0.75 & 0.5 & 0.25
\end{array}\right] \\
& \mu=\begin{array}{c}
x_{1} \\
f_{1} \\
f_{2} \\
f_{3} \\
f_{4} \\
f_{5}
\end{array}\left[\begin{array}{cccc}
1 & 0.67 & 0.24 & 0.54 \\
0.64 & 1 & 0.80 & 0.89 \\
1 & 1 & 1 & 0.30 \\
0.30 & 0.43 & 0.43 & 1 \\
0.25 & 0.33 & 0.50 & 1
\end{array}\right]
\end{aligned}
$$

Obviously,

$$
\max _{1 \leq j \leq 4} \min _{1 \leq i \leq 5}\left\{\mu_{i j}\right\}=\max \{0.25,0.33,0.24,0.30\}=0.33=\mu_{52}
$$

Therefore, the scheme 2, in which the power of the engine is "2082 Horsepower", the maximum rate of climbing is "750 meter per minute" and the capability of the system is "Strong", the research cost is "Middle" and the research time is "Long", is judged to be the optimal scheme. Similarly, the ranking of the schemes is "Scheme 2", "Scheme 4", "Scheme 1 " and "Scheme 3".

3) Minimax method of optimizing with different weights

The method of optimizing above doesn't involve weights. However, most of problems are related to several factors [10]. For this reason, we introduce different weights to reflect the different emphasizes. The membership function could be defined as:

$$
\mu_{i^{*} j^{*}}=\max _{1 \leq j \leq n} \min _{1 \leq i \leq m}\left\{\mu_{i j}^{\omega_{i}}\right\}
$$

Therefore, the method, with the preference of each objective could be described, is called minimax method of optimizing with different weights [11]. Assume that the weight vector is

$$
\omega=(0.1,0.2,0.4,0.1,0.2)^{T}
$$

Then, we can get:

$$
\max _{1 \leq j \leq 4} \min _{1 \leq i \leq 5}\left\{\bar{\mu}_{i j}\right\}=\max \{0.76,0.80,0.87,0.62\}=\bar{\mu}_{53}
$$

Therefore, the scheme 3 , in which the power of the engine is "760 Horsepower", the maximum rate of climbing is "600 meter per minute" and the capability of the system is "Strong", the research cost is "Middle" and the research time is "Middle", is judged to be the optimal scheme. Similarly, the ranking of the schemes is "Scheme 3", "Scheme 2", "Scheme 1", and "Scheme 4".

Obviously, the final result given by "Minimax Method of Optimizing with Different Weights" is different from those given by the method without weights.

\section{ANALYSIS OF RESULTS}

According to the operational effectiveness evaluation results, an optimal solution is got by analyzing multiple decision-making combinations which meet the effectiveness performance grades requirements with rough set theory. First, this paper adopted the minimax method and found that the second decision-making scheme combination was the optimal solution. Finds the optimal solution is the second decision-making scheme combination. After that the decision maker who combined their own preference and weights, used the minimax weight method again, getting the third decision scheme combination as the optimal solution. To further analyze different optimization results, the comparison result of scheme 2 and scheme 3 is shown in Table V.

According to Table $\mathrm{V}$, scheme 2 and scheme 3 differ slightly in "the maximum rate of climb", "the platform system" and "the development costs". However, there are significant differences between "power" and "the development time". Scheme 2 is lower than scheme 3 in "power" value and scheme 2 has longer "development time". Thus, if optimization weight is not introduced, in the 
"power", scheme 2 has obvious advantages in the numerical solution over scheme 3 . The final optimization result shows that scheme 2 is the optimal solution. When optimization weight is introduced, as decision makers would pay more attention to "the development time" in which scheme 3 is one level shorter than scheme 2 in length, the optimization result after weighted indicates that the scheme 3 is the optimal solution.

TABLE V: DIFFERENT SCHEMES

\begin{tabular}{|c|c|c|c|}
\hline $\begin{array}{l}\text { schemes } \\
\text { attributes }\end{array}$ & Scheme 2 & Scheme 3 & coefficient \\
\hline Power & 2082 & 760 & 0.1 \\
\hline Rate of climbing & 750 & 600 & 0.2 \\
\hline System & Strong & Strong & 0.4 \\
\hline Cost & Middle & Middle & 0.1 \\
\hline Time & Long & Middle & 0.2 \\
\hline
\end{tabular}

\section{CONCLUSION}

This paper proposes an effectiveness evaluation method based on rough set theory and takes the performance index set of top 20 gunships around the world nowadays as the knowledge base. First, this paper evaluates the operational effectiveness of decision-making based on various performance indicators combination and gets decision schemes satisfying the completion degree requirements after filtering. The decision schemes filtered are then solved according to the multi-objective decision optimization method. Experiments are done both before and after introducing the optimization weight to get different optimal decisions in different conditions with different emphasis. The screening stage is added in the experiment process, to some extent, it reduced the complexity of the follow-up process Correction parameters are also added in the effectiveness evaluation completion confirmation, which could be helpful to get the rough evaluation result. The paper generally realizes the combined application between the rough set theory and multi-objective decision-making optimization in the operational effectiveness evaluation.

\section{REFERENCES}

[1] Z. Pawlak, "Rough sets," International Journal of Computer and Information Science, vol. 11, pp. 341-356, 1982.

[2] Z. M. Li, P. D. Xu, C. X. Ran, and J. Liu, Theory and Application of the Effectiveness of Weapon System, National Defence Industrial Press, vol. 8, pp. 155-170, 2013.

[3] D. F. Li and P. D. Xu, "Evaluation and optimum seeking methods of combat plans with general preference structure," Fuzzy System and Mathematics, vol. 13, pp. 74-79, 1999.

[4] Steve Crawford, Twenty-first Century Military Helicopters, Popular Science Press, vol. 4, pp. 6-190, 2009.

[5] D.-F. Li and S.-Y. Chen, "A fuzzy optimum seeking method for multiple objective decision making with time series," Systems Engineering and Electronics, vol. 16, pp. 12-18, 1994.

[6] D.-F. Li, Fuzzy Multiobjective Many-Person Decision Makings and Games, National Defence Industrial Press, vol. 4, pp. 58-117, 2003.

[7] B. X. Liu, Rough Set Theory of Analysis and Decision Model, vol. 11, pp. 1-109, 2010.

[8] D. Dubois and H. Prade, "Rough fuzzy sets and fuzzy rough sets," International Journal of General System, vol. 17, pp. 191-208, 1990.

[9] H. X. Li, X. Z. Zhou, T. R. Li, G. Y. Wang, D. Q. Miao, and Y. Y. Yao, Decision Rough Set and Researching Development, Science Press, vol. 11, pp. 62-89, 155-179, 2011.

[10] D. F. Li and S. Y. Chen, "A fuzzy programming approach to fuzzy linear fractional programming with fuzzy coefficients," The Iournal of Fuzzy Mathematics, vol. 4, pp. 829-833, 1996.

[11] Y. J. Feng, "Fuzzy solution of multi objective programming problems," Science Bulletin, vol. 17, pp. 1028-1030, 1981.

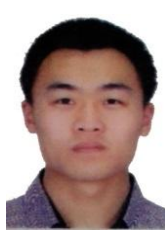

Zhenghua Cui was born in Jilin, China in 1990. He is with the National University of Defense Technology, Changsha, Hunan, China. He has got the academic master degree of engineering. His research area is task planning technology.

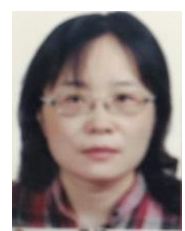

Jian Huang was born in Zhejiang, China in 1973. She is with the National University of Defense Technology, Changsha, Hunan, China. She has got the academic doctor degree of engineering. She is a D.E. Ph.D. supervisor. Her research area is task planning technology. 